Fi ni te El ement Anal ysi s of Nonl i near Magnet i c Devi ces Combi ned wi th Ci r cui t Equat i ons by Tabl eau Appr oach

\begin{tabular}{|l|l|}
\hline 著者 & Yanada Sot oshi, Bessho Kazuo, Ki t agawa Naki ko \\
\hline $\begin{array}{l}\text { j our nal or } \\
\text { publ i cat i on } \mathrm{t} \mathrm{it} \mathrm{l} \mathrm{e}\end{array}$ & I EEE Tr ansact i ons on Nagget i cs \\
\hline vol une & 28 \\
\hline nunber & 5 \\
\hline page r ange & $2256-2258$ \\
\hline year & 1992-09-01 \\
\hline URL & ht t p: //hdl . handl e. net /2297/48302 \\
\hline
\end{tabular}




\title{
FINITE ELEMENT ANALYSIS OF NONLINEAR MAGNETIC DEVICES COMBINED WITH CIRCUIT EQUATIONS BY TABLEAU APPROACH
}

\author{
Sotoshi YAMADA, Kazuo BESSHO, and Masaki KITAGAWA
}

Faculty of Technology, Kanazawa Univer sity, Kodatsuno 2-40-20, Kanazawa, Japan

\begin{abstract}
When simulating electric machines, the magnetic field analysis and the circuit calculation are coupled closely. We present a method in which the combination of Maxwell's equations and circuit equations is solved simultaneously. The tableau approach as nodal formulation is applied to the circuit model. This paper describes a new generalized approach for considering the external networks in the harmonic balance finite element method we proposed. The approach is applied to the analysis of a magnetic frequency tripler with circuit elements.
\end{abstract}

\section{INTRODUCTION}

There is sometimes the necessity to analyze electric machines including the external magnetizing circuit. But the consideration of the magnetizing circuit in the field analysis often suppresses the flexibility of the FEM analysis [1], [2]. Meanwhile a previous paper has reported that zero-dimensional finite elements could be used to circuit model [3].

The authors developed the harmonic balance finite element method (HBFEM) for the analysis of the time-periodic eddycurrent problems in the nonlinear $\mathrm{AC}$ devices [4]. In the process of HBFEM, it is important to calculate both the field and circuit procedures simultaneously because of nonlinearity. The tableau approach is well-known as nodal formulation in the numerical analysis of the lumped circuit model [5]. We combine HBFEM with the circuit equation expressed by the tableau approach.

\section{HBFEM AND TABLEAU APPROACH}

Let us discuss the generalized model shown in Fig.1. The figure shows the schematic diagram in which the domain of field problems by FEM and the circuit equations for the networks are coupled. In this model, we consider the time-periodic problems with an $A C$ magnetizing source.

HBFEM is the steady-state analysis for the nonlinear dynamic magnetic field problems in the harmonic domain [4]. For time-periodic eddy-current problems with magnetic saturation, the vector potential $A^{i}$ and applied current density $J_{o}$ can be expressed in 2-D Cartesian coordinates as

$$
\begin{aligned}
& A^{i}=\sum_{m=1,3,5 \ldots}\left\{A_{m s}^{i} \sin (m \omega t)+A_{m c}^{i} \cos (m \omega t)\right\} \\
& J_{0}=\sum_{m=1,3,5 \ldots}\left\{J_{m s} \sin (m \omega t)+J_{m c} \cos (m \omega t)\right\}
\end{aligned}
$$

where each variables have odd-order harmonics. The subscripts $\mathrm{s}$ and $c$ denote the sine- and cosine-components and the superscript $i$ indicates the node number.

In order to consider the nonlinear $\mathrm{B}-\mathrm{H}$ curve $H\{B(t)\}$ of a core, we express the magnetic reluctivity during a single cycle as

$$
\nu(t)=\nu_{0}+\sum_{m=2,4,6 \ldots}\left\{\nu_{m s} \sin (m \omega t)+\nu_{m c} \cos (m \omega t)\right\}
$$

where $\nu_{0}, \nu_{m s}$, and $\nu_{m c}$ are the coefficients of the Fourier transformation [4].

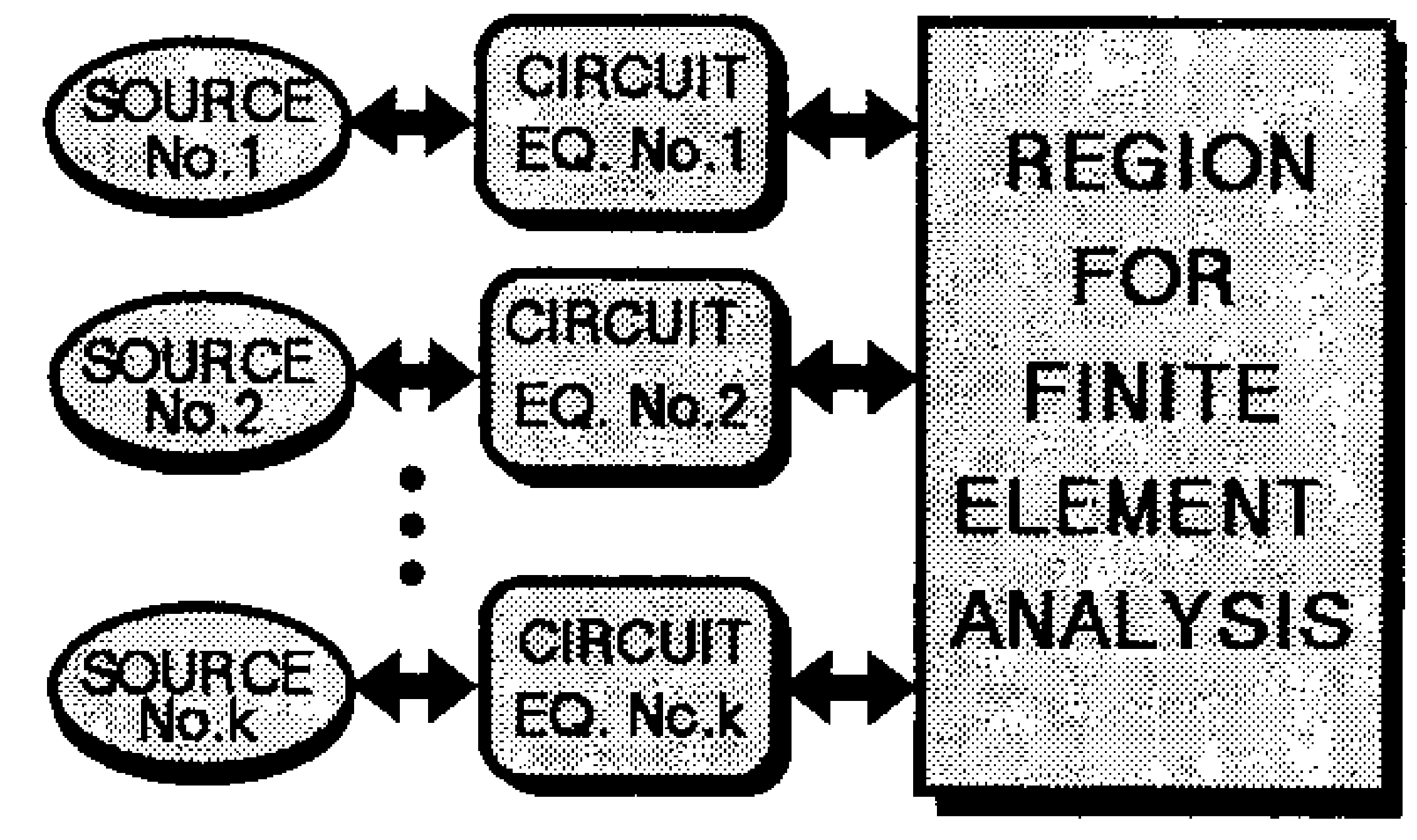

Fig.1 Model with the combination of FEM and circuit equation

Substituting (1)-(3) into the Galerkin's equation, we obtain the matrix equation for a first order triangular element, $e_{1}$ as the coefficients of $\sin (m \omega t)$ and $\cos (m \omega t)$

$$
\begin{aligned}
& \frac{1}{4 \Delta}\left[\begin{array}{lll}
S_{11} \mathbf{D}_{m m} & S_{12} \mathbf{D}_{m m} & S_{13} \mathbf{D}_{m m} \\
S_{21} \mathbf{D}_{m m} & S_{22} \mathbf{D}_{m m} & S_{23} \mathbf{D}_{m m} \\
S_{31} \mathbf{D}_{m m} & S_{32} \mathbf{D}_{m m} & S_{33} \mathbf{D}_{m m}
\end{array}\right]\left\{A_{m}^{e}\right\} \\
& +\frac{\sigma \omega \Delta}{12}\left[\begin{array}{ccc}
2 \mathbf{N}_{m} & \mathbf{N}_{m} & \mathbf{N}_{m} \\
\mathbf{N}_{m} & 2 \mathbf{N}_{m} & \mathbf{N}_{m} \\
\mathbf{N}_{m} & \mathbf{N}_{m} & 2 \mathbf{N}_{m}
\end{array}\right]\left\{A_{m}^{e}\right\} \\
& =-\sum_{j=1,3,5 \ldots}^{j \neq m} \frac{1}{4 \Delta}\left[\begin{array}{ccc}
S_{11} \mathbf{D}_{m j} & S_{12} \mathbf{D}_{m}, & S_{13} \mathbf{D}_{m j} \\
S_{21} \mathbf{D}_{m j} & S_{22} \mathbf{D}_{m j} & S_{23} \mathbf{D}_{m j} \\
S_{31} \mathbf{D}_{m j} & S_{32} \mathbf{D}_{m j} & S_{33} \mathbf{D}_{m j}
\end{array}\right]\left\{A_{j}^{e}\right\}+\left\{K_{m}^{e}\right\}
\end{aligned}
$$

where $\Delta$ is the area of a triangular element And the column vectors $\left\{A_{m}^{e}\right\}$ and $\left\{K_{m}^{e}\right\}$ are given by

$$
\begin{aligned}
& \left\{A_{m}^{\mathrm{e}}\right\}=\left\{\begin{array}{llllll}
A_{m s}^{1} & A_{m c}^{1} & A_{m s}^{2} & A_{m c}^{2} & A_{m s}^{3} & A_{m c}^{3}
\end{array}\right\}^{T} \\
& \left\{K_{m}^{c}\right\}=\frac{\Delta}{3}\left\{\begin{array}{lllllll}
J_{m s} & J_{m c} & J_{m s} & J_{m c} & J_{m s} & J_{m c}
\end{array}\right\}^{T}
\end{aligned}
$$

The superscripts 1,2 , and 3 denote the node number of a triangular element. The coefficients $S_{i}$ are given in reference [4]. Here, the block matrix $D_{m}$ is the reluctivity matrix describing the nonlinearity of the core, then

$$
\begin{aligned}
\mathbf{D}_{m m} & =\mathbf{D}_{(2 k-1)(2 k-1)} \\
& =\frac{1}{2}\left[\begin{array}{cc}
2 \nu_{0}-\nu_{2(2 k-1) c} & \nu_{2(2 k-1) s} \\
\nu_{2(2 k-1) s} & 2 \nu_{0}+\nu_{2(2 k-1) c}
\end{array}\right] \\
\mathbf{D}_{m s} & =\mathbf{D}_{\left(2 k_{1}-1\right)\left(2 k_{2}-1\right)} \\
& =\frac{1}{2}\left[\begin{array}{cc}
\nu_{2\left(k_{2}-k_{1}\right) c}-\nu_{2\left(k_{1}+k_{2}-1\right) c} & -\nu_{2\left(k_{2}-k_{1}\right) s}+\nu_{2\left(k_{1}+k_{2}-1\right) s} \\
\nu_{2\left(k_{2}-k_{1}\right) s}+\nu_{2\left(k_{1}+k_{2}-1\right) s} & \nu_{2\left(k_{2}-k_{1}\right) c}+\nu_{2\left(k_{1}+k_{2}-1\right) c}
\end{array}\right]
\end{aligned}
$$

The harmonic matrix $\mathbf{N}_{m}$ is expressed by

$$
\mathrm{N}_{m}=\left[\begin{array}{cc}
0 & -m \\
m & 0
\end{array}\right]
$$

The system equation for the entire region of interest is arranged by the conventional FEM procedure [4]. Based on (4), the system 
equations for the fundamental, third, and h-th order components are

$$
\begin{gathered}
{\left[H_{11}\right]\left\{A_{1}\right\}^{k}=-\sum_{\jmath=3,5, \ldots h}\left[H_{1 \jmath}\right]\left\{A_{\jmath}\right\}^{k-1}+\left\{K_{1}\right\}} \\
{\left[H_{33}\right]\left\{A_{3}\right\}^{k}=-\sum_{\jmath=1,5, \ldots h}\left[H_{3}\right]\left\{A_{j}\right\}^{k-1}+\left\{K_{3}\right\}} \\
\left.\vdots H_{h h}\right]\left\{A_{h}\right\}^{k}=-\sum_{\jmath=1,3, \ldots h-1}\left[H_{h}\right]\left\{A_{\jmath}\right\}^{k-1}+\left\{K_{h}\right\}
\end{gathered}
$$

where $\left\{K_{3}\right\}$ is the driving term of the applied current. As higher harmonic components decrease gradually in the physical model, we have the system equations for harmonic components up to h-th order. The first term on the right-hand side means that the harmonic components except the $j$-th order affect the $j$-order harmonic. We apply the iterative calculation process to (9). The superscripts $k$ and $(k-1)$ denote the $k$ - and $(k-1)$-th iterations.

To formulate the magnetizing circuit, we consider the nodal formulation, thus the tableau approach which is known in the circuit analysis [5]. When considering the circuit model with $n$ nodes and $b$ branches, we obtain one matrix equation

$$
\left[\begin{array}{ccc}
\mathbf{I} & \mathbf{0} & -\mathbf{A}_{e}^{T} \\
\mathbf{y} & \mathbf{z} & \mathbf{0} \\
\mathbf{0} & \mathbf{A}_{e} & \mathbf{0}
\end{array}\right]\left\{\begin{array}{c}
\left\{v^{b}\right\} \\
\left\{i^{b}\right\} \\
\left\{v^{n}\right\}
\end{array}\right\}=\left\{\begin{array}{c}
\mathbf{0} \\
\left\{v^{i n}\right\} \\
\mathbf{0}
\end{array}\right\}
$$

This formulation involves three matrix equations, the Kirchhoff's voltage law, the constitution equations, and the Kirchhoff's current law in reference [5]. The column vectors $\left\{v^{b}\right\},\left\{v^{n}\right\}$, and $\left\{i^{b}\right\}$ include all branch voltages, node voltages, and branch currents. $\mathbf{A}_{e}$ is the $(n-1) \times b$ incident matrix. The column vector $\left\{v^{\text {in }}\right\}$ consists of voltage sources and the induced voltage. The matrices $z$ and $y$ are the impedance and admittance.

In order to combine these expressions with HBFEM, we formulate the tableau approach for time-periodic solution. The voltage and current at branches are expressed by

$$
\begin{aligned}
i(t) & =\sum_{m=1,3,5 \ldots}\left\{I_{m s} \sin (m \omega t)+I_{m c} \cos (m \omega t)\right\} \\
v(t) & =\sum_{m=1,3,5 \ldots}\left\{V_{m s} \sin (m \omega t)+V_{m c} \cos (m \omega t)\right\}
\end{aligned}
$$

Hence the equation for a resistor with resistance $R_{k_{k}}$ in the branch $k_{b}$ is given by

$$
\left\{\begin{array}{l}
V_{m s} \\
V_{m c}
\end{array}\right\}=\left[\begin{array}{cc}
R_{k_{s}} & 0 \\
0 & R_{k_{s}}
\end{array}\right]\left\{\begin{array}{l}
I_{m s} \\
I_{m c}
\end{array}\right\}=\left[R_{k_{s}}\right]\left\{\begin{array}{c}
I_{m s} \\
I_{m c}
\end{array}\right\}
$$

Where the subscript $m$ denotes the $\mathrm{m}$-th order harmonic.

The equations for a capacitor with capacitance $C_{k_{k}}$, and an inductor with inductance $L_{k_{*}}$ are respectively

$$
\begin{aligned}
& \left\{\begin{array}{c}
I_{m s} \\
I_{m c}
\end{array}\right\}=\omega \mathrm{N}_{m}\left[\begin{array}{cc}
C_{k_{b}} & 0 \\
0 & C_{k_{b}}
\end{array}\right]\left\{\begin{array}{l}
V_{m s} \\
V_{m c}
\end{array}\right\}=\left[B_{k_{b} m}\right]\left\{\begin{array}{l}
V_{m s} \\
V_{m c}
\end{array}\right\} \\
& \left\{\begin{array}{l}
V_{m s} \\
V_{m c}
\end{array}\right\}=\omega \mathrm{N}_{m}\left[\begin{array}{cc}
L_{k_{b}} & 0 \\
0 & L_{k_{b}}
\end{array}\right]\left\{\begin{array}{l}
I_{m s} \\
I_{m c}
\end{array}\right\}=\left[X_{k_{b} m}\right]\left\{\begin{array}{c}
I_{m s} \\
I_{m c}
\end{array}\right\}
\end{aligned}
$$

By using (12)-(14), Eq.(10) for the m-th order harmonic can be rewritten in the following form;

$$
\left[\begin{array}{ccc}
\mathbf{I} & \mathbf{0} & -\mathbf{A}_{c}^{T} \\
\mathbf{Y}_{m} & \mathbf{Z}_{m} & \mathbf{0} \\
\mathbf{0} & \mathbf{A}_{c} & \mathbf{0}
\end{array}\right]\left\{\begin{array}{l}
\left\{V_{m}^{b}\right\} \\
\left\{I_{m}^{b}\right\} \\
\left\{V_{m}^{n}\right\}
\end{array}\right\}=\left\{\begin{array}{c}
\mathbf{0} \\
\left\{V_{m}^{i n}\right\} \\
\mathbf{0}
\end{array}\right\}
$$

The relations between the field variables $\left\{A_{m}\right\},\left\{K_{m}\right\}$ and the circuit variables $\left\{I_{m}^{b}\right\},\left\{V_{m}^{i n}\right\}$ are given by

$$
\begin{aligned}
\left\{V_{m}^{m}\right\} & =\mathrm{C}_{m}\left\{A_{m}\right\} \\
\left\{K_{m}\right\} & =\mathrm{G}\left\{I_{m}^{b}\right\}
\end{aligned}
$$

where the generation of the matrices $C_{m}$ and $G$ are given in reference [1]. By combining (9) and (15), the system equation for $\mathbf{m}$-th order harmonic is expressed as

$$
\left[\begin{array}{cccc}
{\left[H_{m m}\right\}} & 0 & -\mathrm{G} & 0 \\
0 & \mathrm{I} & 0 & -\mathbf{A}_{c}^{T} \\
-\mathrm{C}_{m} & \mathrm{Y}_{m} & \mathrm{Z}_{m} & 0 \\
0 & 0 & \mathrm{~A}_{e} & 0
\end{array}\right]\left\{\begin{array}{c}
\left\{A_{m}\right\}^{k} \\
\left\{V_{m}^{b}\right\}^{k} \\
\left\{I_{m}^{b}\right\}^{k} \\
\left\{V_{m}^{n}\right\}^{k}
\end{array}\right\}=\left\{\begin{array}{c}
\left\{F_{m}\right\} \\
\{0\} \\
\left\{V^{i n}\right\} \\
\{0\}
\end{array}\right\}
$$

where $\left\{V^{n}\right\}$ is the voltage source and $\left\{F_{m}\right\}$ is given by

$$
\left\{F_{m}\right\}=-\sum_{j=1,3, \ldots h}^{j \neq m}\left[H_{m j}\right]\left\{A_{j}\right\}^{k-1}
$$

The HBFEM system equation for the whole magnetic field and circuit equation are obtained.

\section{ANALYSIS OF MAGNETIC FREQUENCY TRIPLER}

We apply the HBFEM to the magnetic frequency tripler with five-legged core shown in Fig.2. This is a typical model of a nonlinear magnetic device with circuit elements at both the input and output sides.

This device contains a five-legged core, three magnetizing coils and two secondary coils connected in series as an output. The magnetic legs with the $u$-, v-, and w-phase windings operate in saturation level and the third harmonic voltage, one of the gen-

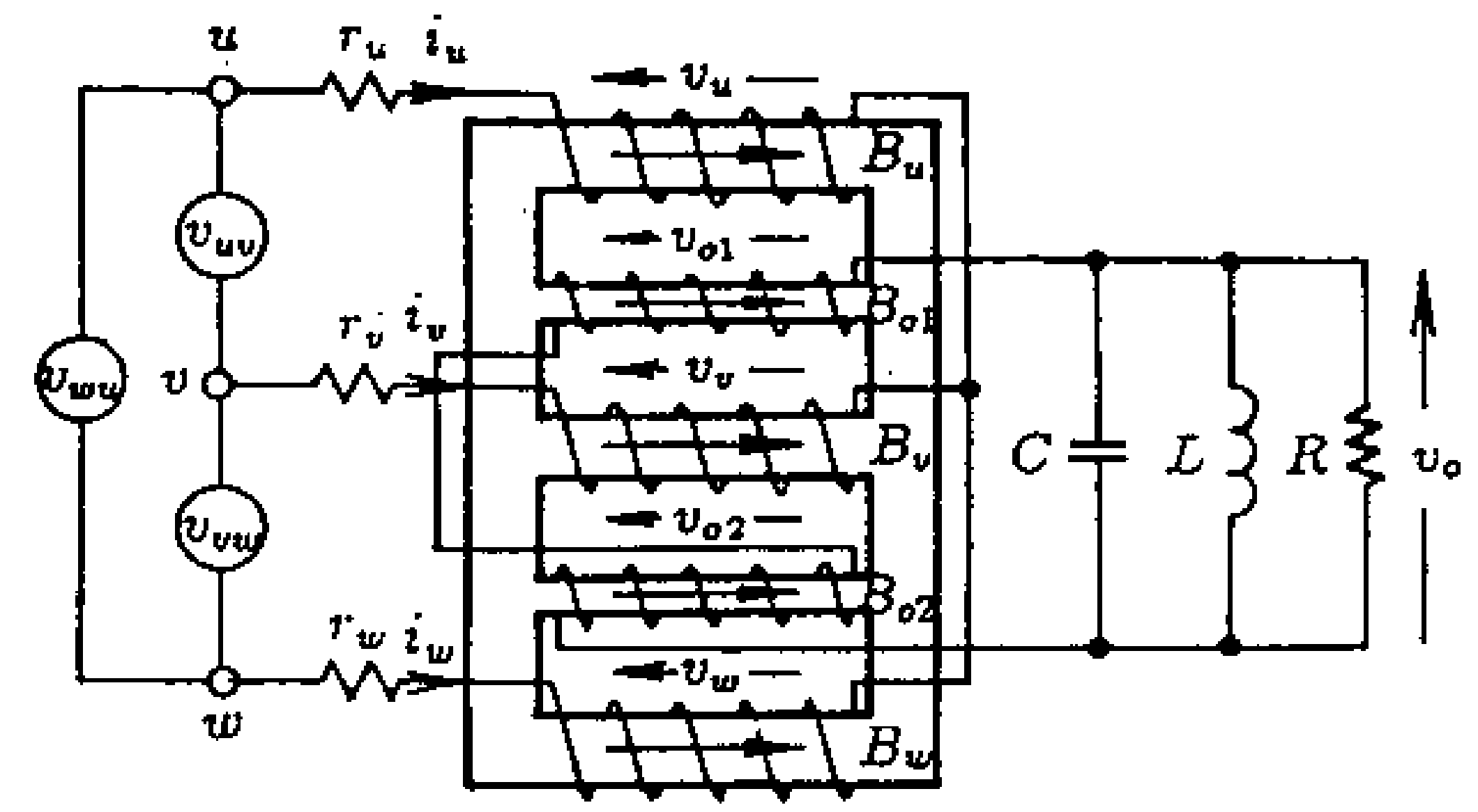

Fig.2 Magnetic frequency frequency tripler

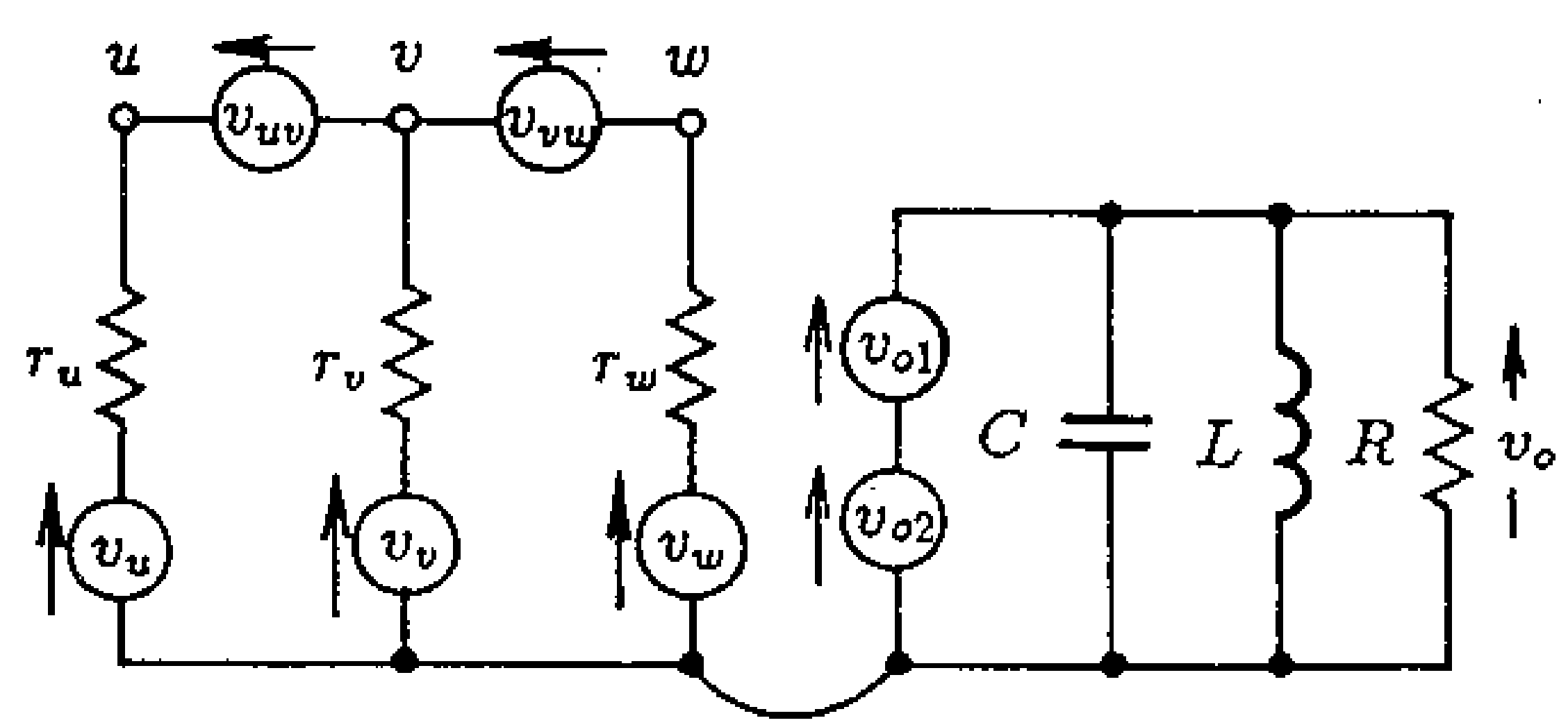

(a) Network

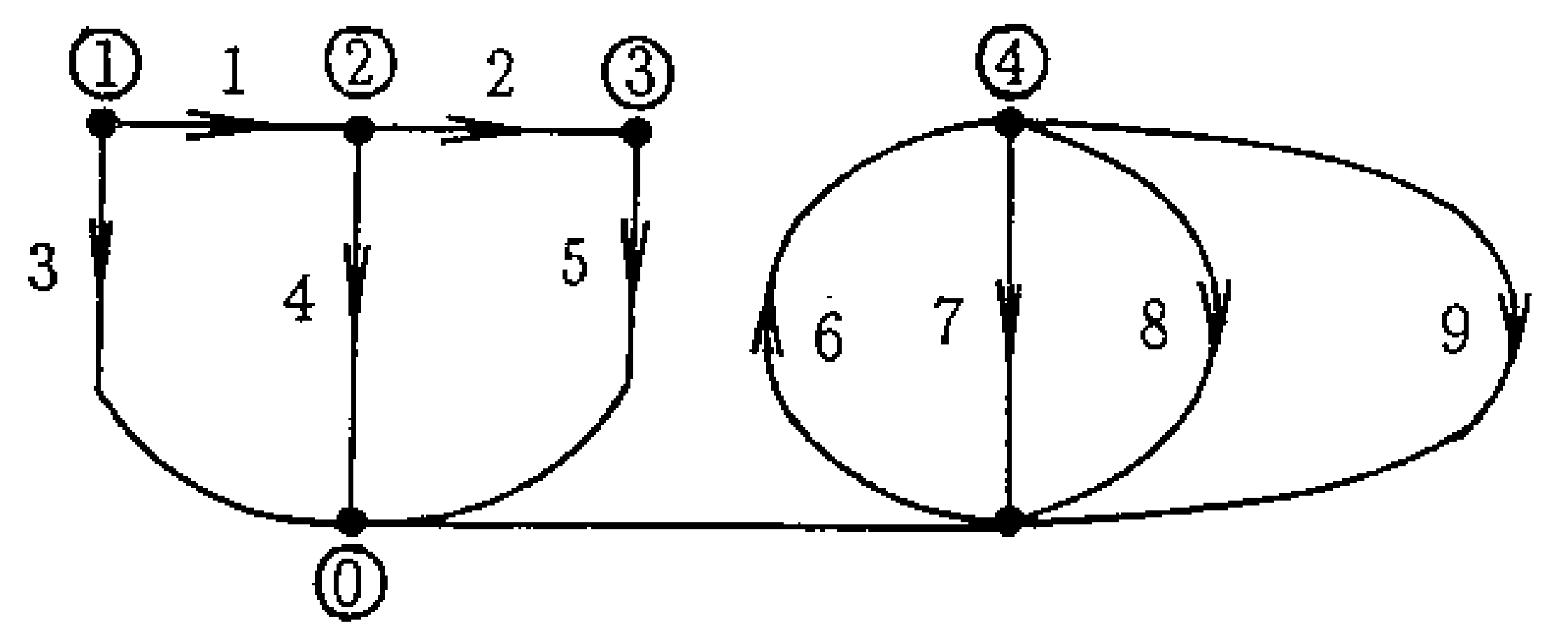

(b) Oriented graph

Fig. 3 Network model for the tripler 
erating harmonics, is produced as output. The half of the cross section of the magnetic core is the analytical domain for the 2-D HBFEM. For simplicity of calculation, the hysteresis of the core is neglected and the $\mathrm{B}-\mathrm{H}$ curve is approximated by

$$
H(B)=100 B+40 B^{9}
$$

Figure 3 shows the circuit network at both the input and output sides. The node and branch are numbered in the graph as shown in Fig.3(b). The voltages $V_{u}, V_{v}$, and $V_{w}$ are the induced voltages at an input side. $V_{o 1}$ and $V_{o 2}$ are the secondary winding voltages. The matrices $A_{\varepsilon}, Y_{m}$ and $Z_{m}$ are given by

$$
\begin{aligned}
& A_{c}=\left[\begin{array}{ccccccccc}
I & & I & & & & & \\
-I & I & & I & & & & & \\
& -I & & I & & & & \\
& & & & -I & I & I & I
\end{array}\right]
\end{aligned}
$$

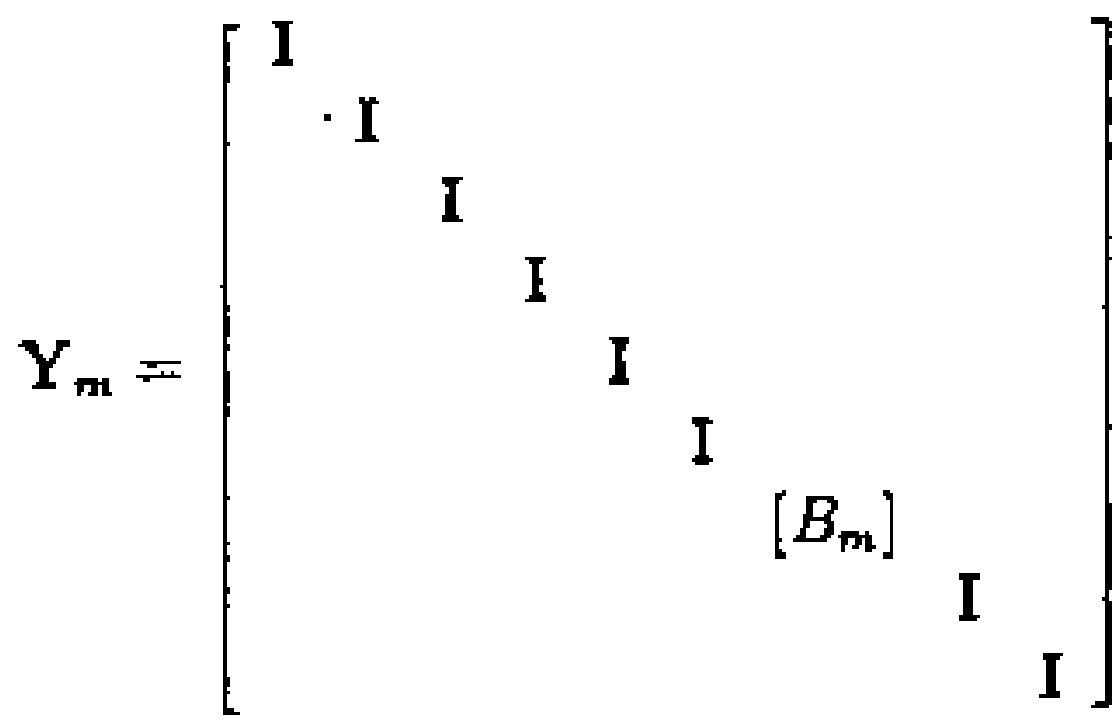

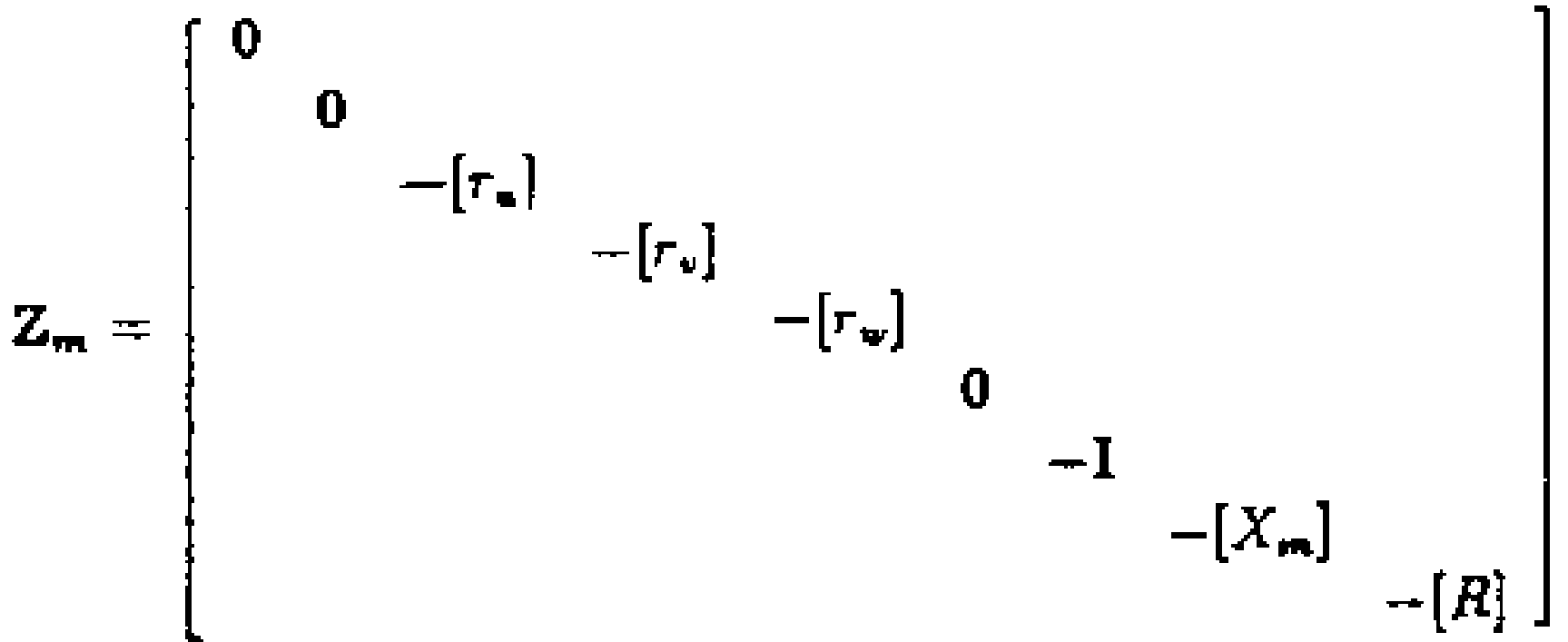

where $I$ is the $2 \times 2$ unit matrix. The column vectors are given by

$$
\begin{aligned}
& \begin{array}{l}
\left\{A_{m}\right\}=\left\{\begin{array}{lllllllll}
A_{m s}^{1} & A_{m c}^{1} & A_{m s}^{2} & A_{m c}^{2} & \ldots & A_{m s}^{2} & A_{m c}^{z} & \ldots
\end{array}\right\}^{T} \\
\left\{V_{m}^{b}\right\}=\left\{\begin{array}{lllllll}
V_{b, m s}^{1} & V_{b, m c}^{1} & V_{b, m s}^{2} & V_{b, m c}^{2} & \ldots & V_{b, m s}^{9} & V_{b, m c}^{9}
\end{array}\right\}^{T}
\end{array} \\
& \left.\left\{I_{m}^{b}\right\}=\begin{array}{lllllll}
I_{b, m s}^{1} & I_{b, m c}^{1} & I_{b, m s}^{2} & I_{b, m c}^{2} & \ldots & I_{b, m s}^{9} & I_{b, m c}^{9}
\end{array}\right\}^{T} \\
& \left\{V_{m}^{n}\right\}=\left\{\begin{array}{lllllll}
V_{n, m s}^{1} & V_{n, m c}^{1} & V_{n, m s}^{2} & V_{n, m c}^{2} & \cdots & V_{n, m s}^{4} & V_{n, m c}^{4}
\end{array}\right\}^{T}
\end{aligned}
$$

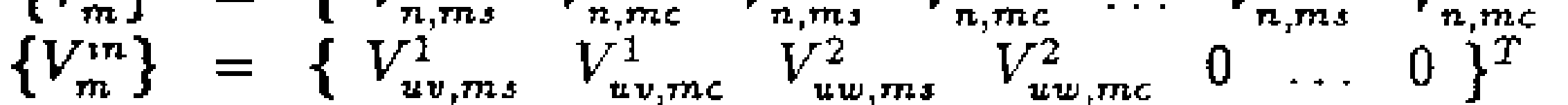

Figure 3 shows the flux distributions for each harmonics up
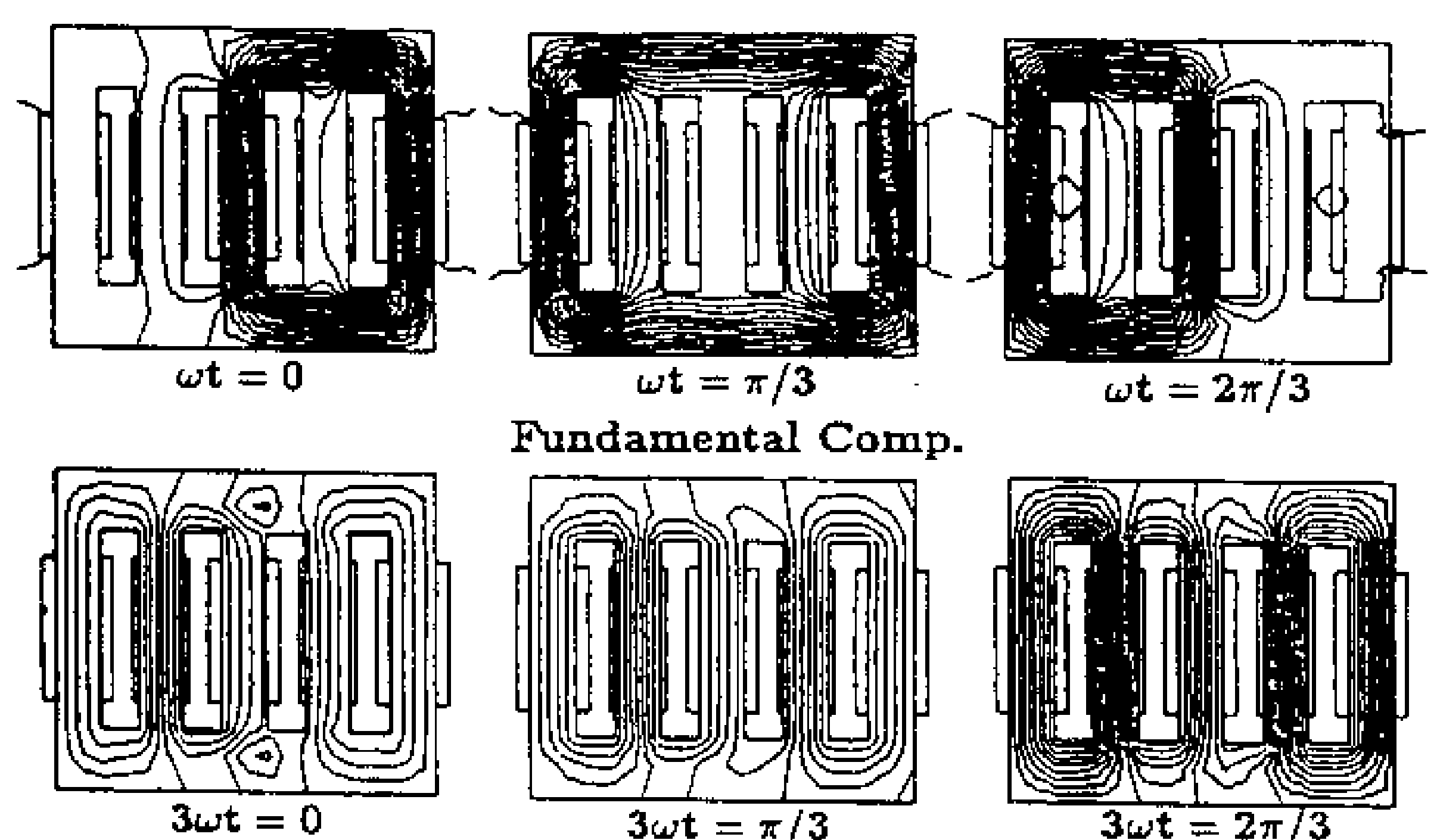

Fundamental Comp.
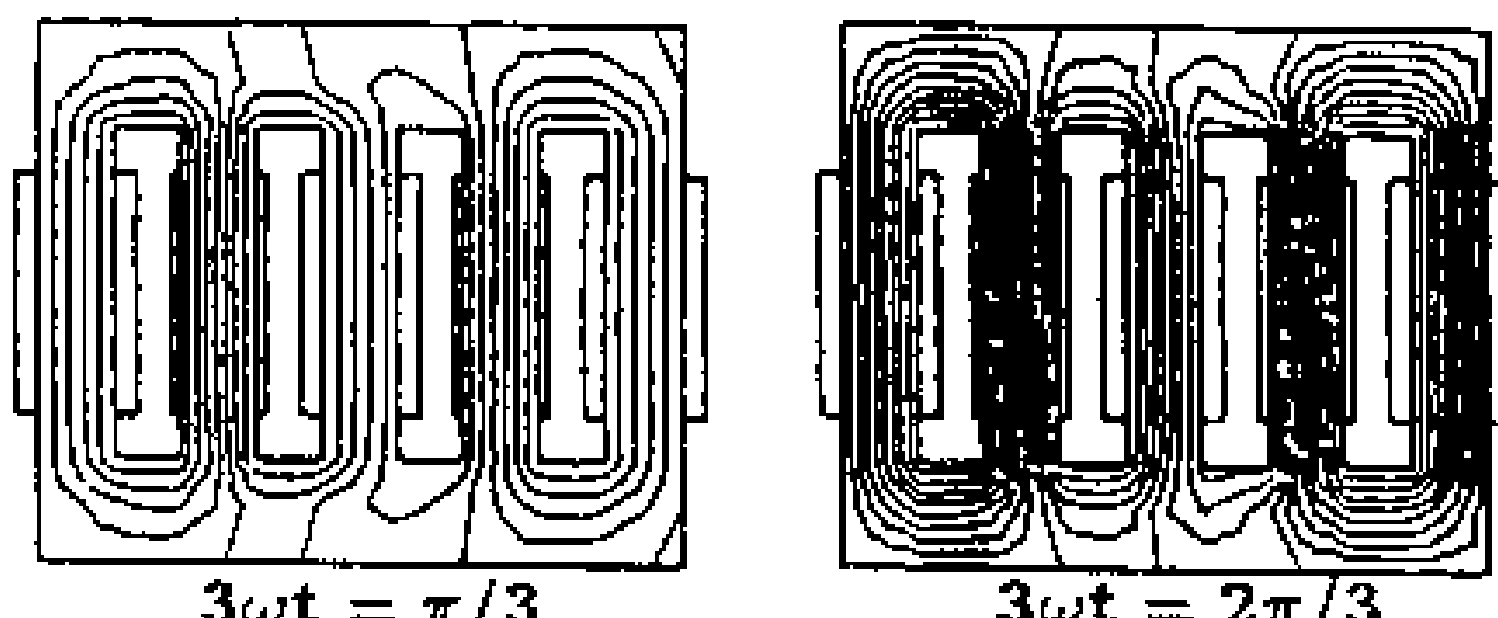

Third Harmonic
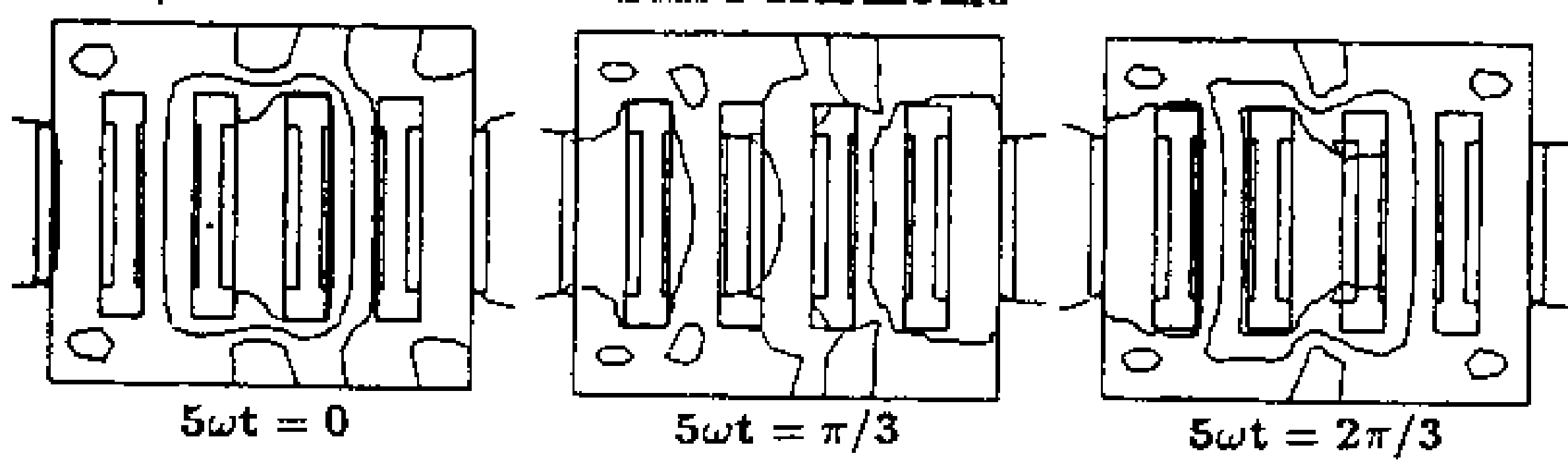

Fifth Harmonic

Fig.4 Flux distribution for fundamental, third and fifth harmonics to 5-th order in the five-legged core. The waveforms of the flux densities and the circuit variables are shown in Fig.4. The results show that the fundamental and thrid harmonic are the major components.

\section{CONCLUSIONS}

This paper proposed the system equation for HBFEM taking into account both the field problem and the circuit equation simultaneously. The formulation of the tableau approach is identical to that of FEM and then the generation of the whole system equation is very easy. The approach provides a powerful tool for the magnetic field problems coupled with circuit equations.

\section{AKNOLEDGEMENTS}

This work was supported in part by BETSUKAWA FOUNDATION.

\section{REFERENCES}

[1] J.Lu, S.Yamada, K.Bessho, "Harmonic Balance Finite Element Method Taking into Account of External Circuit and Motion", IEEE Trans. on Magnetics, Vol.27, No.5, pp.40244027, 1991.

[2] E.Vassent, G.Meunier, A.Foggia, "Simulation of Induction Machines Using Complex Magnetodynamic Finite Element Method Coupled with the Circuit Equations", IEEE Trans. on Magnetics, Vol.27, No.5, pp.4246-4248, 1991.

[3] J.R.Brauer, B.E.MacNeal, L.A.Larkin, V.D.Overbye, "New Method of Modeling Electromagnetic Circuit Coupled with 3D Electromagnetic Finite Element Models", IEEE Trans. on Magnetics, Vol.27, No.5, pp.4085-4088, 1991.

[4] S.Yamada, P.P.Biringer, K.Bessho, "Calculation of Nonlinear Eddy-current Problems by the Harmonic Balance Finite Element method", IEEE Trans. on Magnetics, Vol.27, No.5, pp.4122-4125, 1991.

[5] J.Vlach, K.Singhal, Computer Method for Circuit Analysis and Design, Van Norstrand Reinhold, Ch.4, USA.
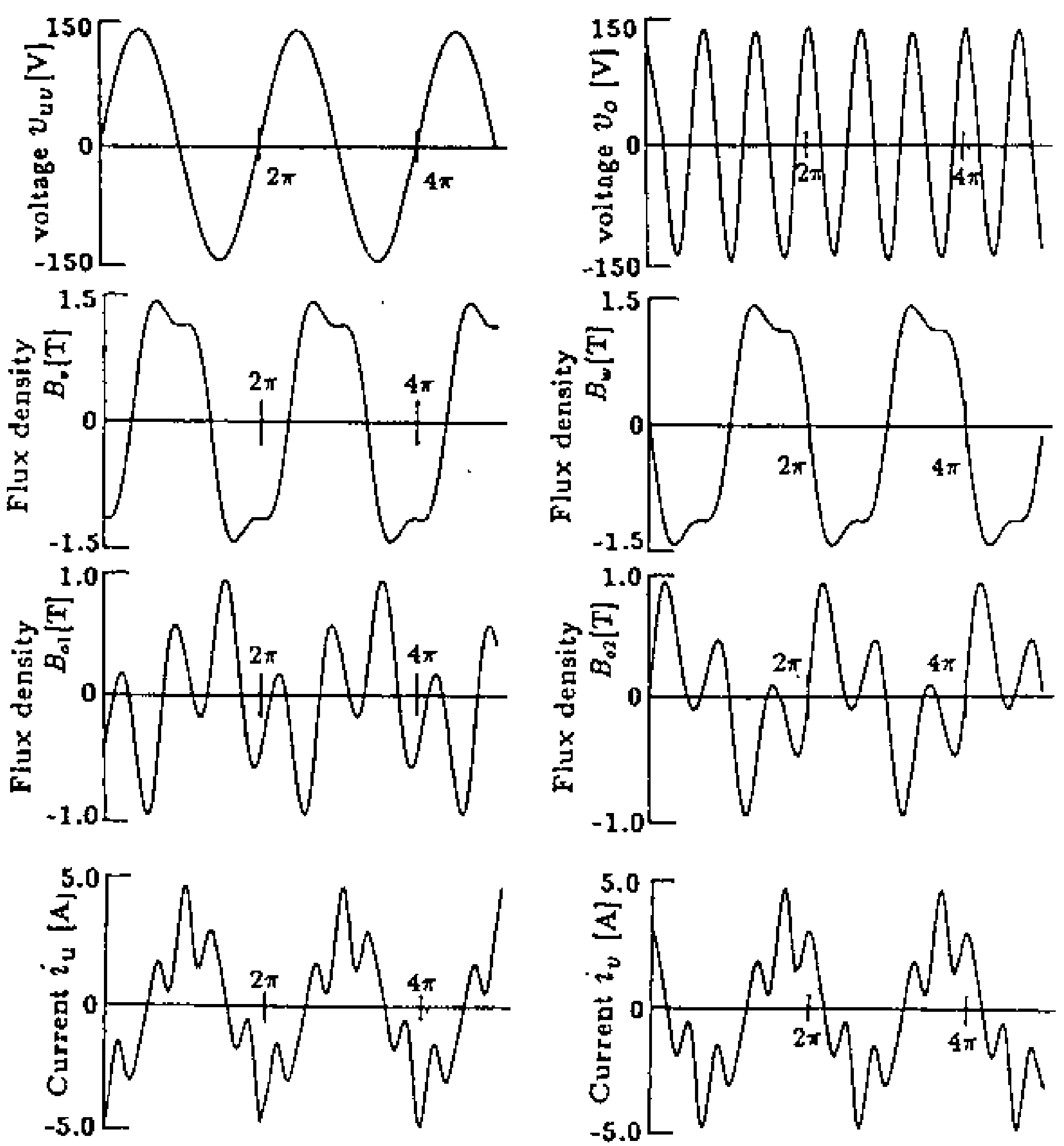

Fig.5 Waveforms of the flux densities, currents and voltages 